\title{
Nicht alkoholische Fettleber: Therapie weiter unklar
}

\author{
Auf der Suche nach einer Thera- \\ piemöglichkeit für die nicht alkoho- \\ lische Steatohepatitis (NASH) haben \\ weder Vitamin E noch Pioglitazon \\ einen überzeugenden Effekt auf die \\ zelluläre Infiltration, Fibrosescores \\ und Transaminasen.
}

— Ein Effekt der Adipositaswelle, die gegenwärtig über die Welt rollt, wird häufig übersehen: die nicht alkoholische Steatohepatitis. Typische histologische Merkmale sind eine Leberzellverfettung und eine Läppcheninfiltration mit Entzündungszellen. Bei bis zu 15\% der betroffenen Patienten schreitet die Erkrankung bis zur Leberzirrhose fort.

Nachdem die Krankheit mit Parametern des metabolischen Syndroms wie Insulinresistenz und Typ-2-Diabetes assoziiert ist, besitzen sowohl Insulinsensitizer wie die Thiazolidinedione und Antioxidantien wie Vitamin E eine potenzielle therapeutische Wirksamkeit.

Der Effekt dieser beiden Substanzen wurde nun bei 247 Erwachsenen mit NASH ohne Diabetes mellitus in einer dreiarmigen randomisierten Studie untersucht. 80 Personen erhielten täglich $30 \mathrm{mg}$ Pioglitazon, 84 Patienten Vitamin $\mathrm{E}$ in einer Dosierung von $800 \mathrm{IU}$ täglich bzw. Placebo (83 Patienten).

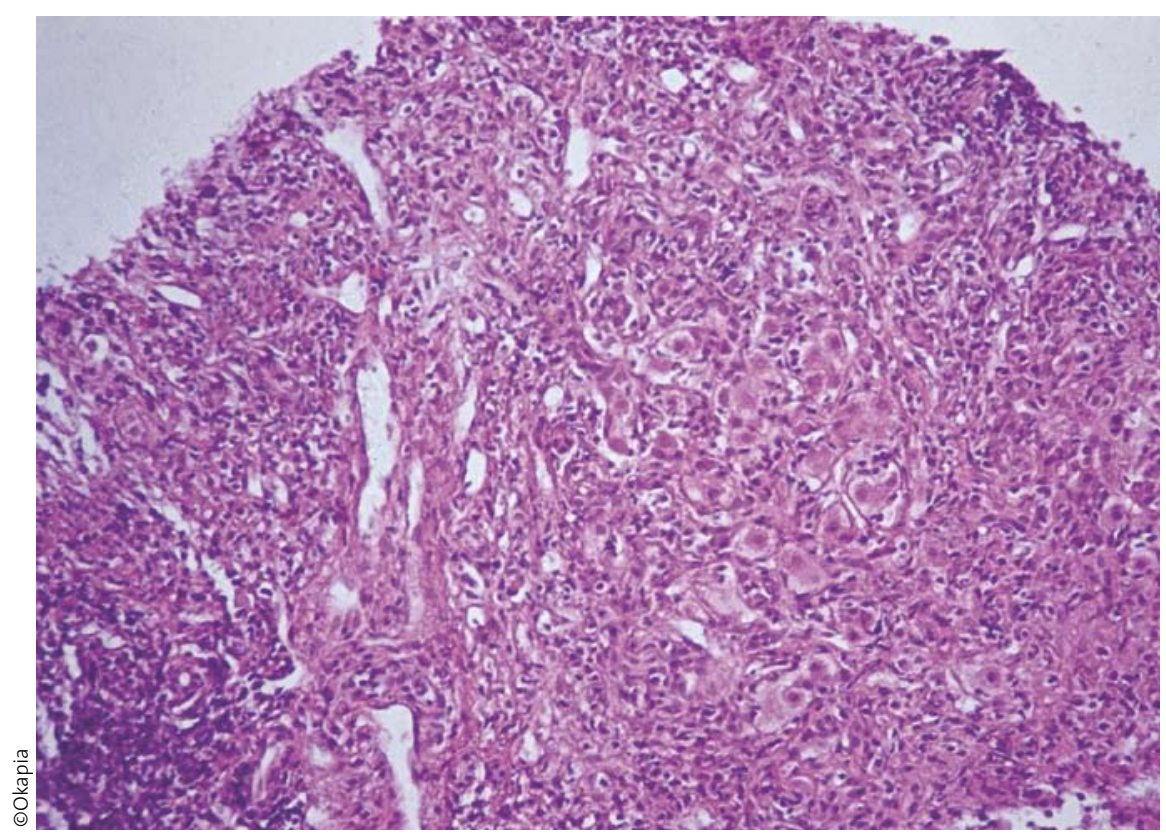

15\% aller Patienten mit NASH entwickeln eine Leberzirrhose.

Die Behandlungsdauer betrug 96 Wochen.

Alle Studienteilnehmer unterzogen sich in den sechs Monaten vor der Randomisierung einer Leberblindpunktion, Patienten mit Leberzirrhose, Hepatitis $\mathrm{C}$ oder anderen Lebererkrankungen, die zu einer Steatohepatitis führen, wurden ausgeschlossen. 90\% der Studienteilnehmer konnten nach der Beendigung der Studie erneut biopsiert werden.

\section{Kommentar}

Bislang gibt es zur Therapie der nicht alkoholischen Steatohepatitis überwiegend vorläufige Erkenntnisse, die in kleinen und zeitlich limitierten Studien gewonnen wurden. Dazu gehören die Untersuchung von Faktoren des Lebensstils, der bariatrischen Chirurgie, regelmäßigen Aderlässen und einer ganzen Reihe von Medikamenten. Aus dieser Konstellation wird klar, dass es die etablierte Therapie der NASH bislang nicht gibt. Die hier vorgelegte Studie untersucht die Effekte von Vitamin $E$ und Pioglitazon auf eine Reihe von Markern für den Leberparenchymschaden, die in uneinheitlicher Weise reagieren. Nachdem viele Parameter herangezogen wurden, wird man das Gefühl nicht los, dass einige der Ergebnisse schlichtweg durch Zufall aufgrund der großen Zahl zustande gekommen sind. Die Studie war außerdem nicht dazu angelegt, den relativen Effekt von Vitamin E und Pioglitazon zu untersuchen. Insgesamt erscheint die optimale Therapie der NASH noch nicht gefunden, wenn man einmal von der Gewichtsreduktion absieht. Über diesen Weg wird aber aufgrund der frustrierenden Ergebnisse offensichtlich kaum noch gesprochen.
Als Zielparameter betrachtete man eine Besserung der histologischen Befunde um wenigstens einen Punkt auf der histologischen Klassifikationsskala, die eine Vakuolisierung der Leberzellen, den Fibrosegrad und die zelluläre Infiltration beinhaltete. In der Vitamin-EGruppe erreichten 43\%, in der Pioglitazongruppe 34\% der Probanden diesen Endpunkt, allerdings auch 19\% unter Placebo. Der Unterschied zwischen Placebo und Vitamin E war hochsignifikant, nicht dagegen zwischen Pioglitazon und Placebo. Die Transaminasen gingen unter beiden aktiven Therapieformen deutlicher zurück als unter Placebo, ebenso die entzündliche Infiltration der Leberläppchen, nicht jedoch der Fibrosegrad. Zudem nahmen die Probanden unter Pioglitazon mehr Gewicht zu als unter Vitamin E oder Placebo.

H. S. FüEßL =

\footnotetext{
- A. J. Sanyal et al.

(Korr.: Arun J. Sanyal, MD, MCV Box 980342, Richmond, VA 23298-0342, e-mail: asanyal@ mcvh-vcu.edu): Pioglitazone, vitamin E, or placebo for nonalcoholic steatohepatitis. New Engl. J. Med. 362 (2010) 18, 1675-1685
} 